\title{
CONTINUOUS-TIME DYNAMIC PRICING FOR STABILIZING STOCHASTIC DEMAND
}

\author{
Kimitoshi Sato \\ Kanagawa University
}

\author{
Katsushige Sawaki \\ Nantong University / CRISER*
}

(Received February 29, 2016; Revised December 27, 2016)

\begin{abstract}
In this paper, we consider the pricing decision of a retailer who experiences peak demand for a product during a given time interval and wishes to stabilize the demand by adjusting the sales price. The stabilization of demand brings about desirable outcomes such as a reduction in the need for capacity investment and improves the production efficiency in the supply chain. We establish a continuous-time model to analyze the effect of dynamic pricing on peak demand. We find that a closed-form optimal pricing policy minimizes the difference between the actual demand and target level. It is shown that the dynamic pricing not only reduces peak demand but also mitigates fluctuations in the peak demand. Using electricity consumption data as a case study, we show that the proposed pricing policy is effective for reducing the mean peak demand compared to a constant pricing policy.
\end{abstract}

Keywords: Stochastic optimization, dynamic pricing, optimal control, demand stabilization

\section{Introduction}

In this paper, we consider a firm that is selling a non-storable product. The product is sold to customers, and demand for the product exhibits a peak within a specified time interval. The firm has the market power to set the price of the product based on the material price, and to determine the price dynamically so as to mitigate demand fluctuations over the selling period.

The motivation for our study is the operations of an electricity provider. Fuel is procured to generate electricity and electricity prices are determined based on the fuel price. The demand for electricity during afternoon hours in July and August in Japan can be more than double to the early-morning with low demand. This is described as peak demand. Providing the infrastructure to supply large quantities of electricity for short periods during peak demand leads to increased generation and network costs. In addition, this peak may result in a supply shortage due to the capacity constraints on the electricity generation. Recently, advances in smart metering technologies have made it possible to track demand and adjust the pricing level rapidly. Dynamic pricing is one of the mechanisms that could be used for mitigating the effect of demand uncertainties and dampening peak demand by allowing consumers to react to the selling price.

Many researchers have focused on the dynamic pricing of electricity in recent years. For instance, Yousefi et al. [1] and Doostizadeh and Ghasemi [2] provide a day-ahead electricitypricing model to maximize retailer's profits based on the day-ahead energy market prices while considering demand elasticity and consumers' benefit. Roozbehani et al. [3] propose total customer utility minus the supplier's cost of meeting the electricity demand as a dynamic

\footnotetext{
${ }^{*}$ Chubu Region Institute for Social and Economic Research
} 
pricing policy that can potentially maximize social welfare. These studies are formulated in a discrete-time setting and demand stabilization is considered under the objective of profit maximization. However, the discrete-time method is not sufficiently flexible to increases in demand at sub-hourly timescales. A sudden increase in demand at sub-hourly timescales would have undesirable economic consequences and would compromise energy security. For this reason, demand stabilization methods need to be continuous in time. In this paper, we propose a continuous-time model to changes in demand, that is, demand uncertainty. We consider not only stabilizing the demand but also reducing the variance of the selling price. This is because customers of highly public products are not happy with large fluctuations in the price. The optimal price is determined in such a way that the demand and the price are maintained as close to the target demand level and the target price, respectively, as possible. The target price is based on the material price, and deviations in the demand level and the price from their target levels are penalized as costs. Thus, we find an optimal price so as to minimize the total penalty costs. Then we investigate the analytical properties of a dynamic pricing policy and the effectiveness of the policy for modulating peak demand.

The dynamic pricing model can be formulated in terms of stochastic optimal control theory. Stochastic optimal control theory is widely used in finance, economics, maintenance, and production planning to solve a variety of problems. In particular, many authors have adopted it for production planning in manufacturing systems (Bensoussan et al. [4], Sethi and Thompson [5]). Based on such studies, we model a continuous-time dynamic pricing problem in terms of stochastic demand, and obtain a closed-form optimal price with the probability distribution of peak demand determined under the dynamic pricing. By comparing our model with a fixed pricing policy, we find that dynamic pricing not only reduces peak demand but also mitigates fluctuations in peak demand. Furthermore, we numerically investigate the impact of consumer's price sensitivity on peak demand.

Our model also has potential applications in other settings. Examples include highly public products (e.g., water, gas, communication networks, and transportation systems) and service products (e.g., the peak occurs in the evening in a supermarket and at lunch time in a restaurant). These industries have the same objective of mitigating demand uncertainty.

The rest of the paper is organized as follows. In Section 2, we present a dynamic pricing model and then develop an optimal pricing policy in Section 3. In Section 4, we present numerical results to evaluate the effect of the proposed pricing policy on demand stabilization. Finally, we discuss our conclusions and future research topics in Section 5. All omitted proofs are given in the appendix.

\section{Dynamic Pricing Model for Demand Stabilization}

We consider a finite horizon problem within time interval $[0, T]$. At the beginning of the selling season, the firm procures some commodities from the market. The firm processes the commodities and sells the resulting product to price-sensitive customers. We assume a peak demand occurs during $[0, T]$ when the product sells at a fixed price $p_{0}$. The peak time is denoted $\hat{t}$. The problem faced by the firm is how to determine an optimal pricing policy so as to stabilize peak demand.

We assume that the demand at time $s, X_{s}$, follows a stochastic differential equation $(\mathrm{SDE})$ :

$$
d X_{s}=\mu(s) d s+\sigma(s) d w_{s}, X_{0}=x,
$$

where $x$ is the initial demand at time $s=0, \mu(s)$ is the growth rate at time $s, \sigma(s)$ is the volatility, and $d w_{t}$ is the random error following a Wiener process. Since the demand has a 
peak at time $\hat{t}$, we define the growth rate as

$$
\mu(s)= \begin{cases}\mu_{1}>0, & \text { if } 0 \leq s<\hat{t} \\ \mu_{2}<0, & \text { if } \hat{t} \leq s \leq \bar{T}\end{cases}
$$

Remark 2.1. For electricity suppliers, demand has the potential to take negative values. For example, an energy supply from renewable sources exceeding the total demand is equivalent to a negative demand. However, occurrences of negative demand are rare for products that are in constant use by many customers (e.g., electricity or communications traffic). Moreover, these demand dynamics allow us to derive the analytical solution for an optimal pricing policy, whereas a general form SDE makes the optimal policy analytically intractable. For this reason, we leave the extension to a more general SDE for future work.

Remark 2.2. We model time-varying demand with a piecewise-linear drift parameter in Equation (2.2). The selling period is divided into two periods $[0, \hat{t}]$ and $[\hat{t}, T]$, and the drift is specified for each sub-period. The low division number would lead to a poor fit of the demand process for actual data. Although we can improve the fit by increasing the division number, the calculus of finding the optimal policy becomes more complex. For this reason, we define the drift parameter with Equation (2.2). Furthermore, the use of a trigonometric function instead of a constant drift would not only retain continuity of the SDE but also lead to a more realistic load profile. We also leave this extension for future work.

In order to derive the relation between the demand and selling price, we introduce a linear price-response function defined by

$$
q\left(s, p_{s}\right)=a_{s}\left(p_{s}-p_{0}\right)+1
$$

where

$$
a_{s}= \begin{cases}a_{1}<0, & \text { if } 0 \leq s<\hat{t} \\ a_{2}=-a_{1}>0, & \text { if } \hat{t} \leq s \leq \bar{T}\end{cases}
$$

The value $a_{s}$ is a price response factor and can be determined by the elasticity $\epsilon$. That is, for any $s \in[0, \hat{t})$, we have

$$
\epsilon\left(p_{0}\right)=-\frac{p_{0} \partial q\left(s, p_{0}\right) / \partial p_{s}}{q\left(s, p_{0}\right)}=-a_{s} p_{0}
$$

giving $a_{s}=a_{1}=-\epsilon / p_{0}$. Thus, the more sensitive the customers are to the price, the larger $\left|a_{s}\right|$ becomes. Using the function $q(\cdot, \cdot)$, the demand under dynamic pricing is given by ${ }^{\dagger}$

$$
d X_{s}=\mu(s) q\left(s, p_{s}\right) d s+\sigma(s) d w_{s} .
$$

Note that if the price $p_{s}$ is larger than the historical average price $p_{0}$, the growth rate of the demand process decreases before the peak. If $p_{s}=p_{0}$ for all $s$, then the demand dynamics

\footnotetext{
†The stochastic part of the demand process in Equation (2.1) accounts for demand increases or decreases that are random in nature. The mean demand is given by $\mu_{i} t, i=1,2$, and the noise term shifts the demand randomly about this mean. Although the process governing the demand of a product can depend on variables other than its price, we focus on the demand process that depends only on current price. In other words, we assume that the price only affects the mean but not the random fluctuations in the demand. Thus, the demand process is controlled by changing the growth rate with the price-response function.
} 
of (2.6) are consistent with (2.1), because $q\left(s, p_{s}\right)=q\left(s, p_{0}\right)=1$. Thus, with the function $q$, we can influence the demand by adjusting the selling price $p_{s}$ at time $s$.

We now define the additional costs incurred by deviating from the target demand level $\theta$ and from the selling target price $\bar{p} .{ }^{\ddagger}$ Here, $\theta>0$ and $\bar{p}>0$ are specified at time 0 and do not change. The target value $\theta$ is determined by the firm on the basis of supply capacity. In addition, the firm determines the target selling price $\bar{p}$ based on the commodity price. Given a demand level $x$ and selling price $p$, the associated total expected cost is

$$
\psi_{s}(p, x)=E\left[\int_{s}^{T}\left\{\pi_{1}\left(X_{u}-\theta\right)^{2}+\pi_{2}\left(p_{u}-\bar{p}\right)^{2}\right\} d u \mid X_{s}=x\right]
$$

where $\pi_{1}$ and $\pi_{2}$ are stability factors. Then, our problem is to optimize the pricing policy by minimizing the function $\psi_{s}(\cdot, \cdot)$ :

$$
\Psi_{s}(x)=\min _{p} \psi_{s}(p, x)
$$

where, at the maturity $T$, Equation (2.7) can be reduced to $\Psi_{T}(x)=\pi_{1}(x-\theta)^{2}$ as the boundary condition at $T$, because the demand is only to be stabilized to within the target level $\theta$. This type of problem has been studied as a linear quadratic optimal control problem (Yong and Zhou [7]).

\section{Optimal Pricing Policy}

The optimal value of $\Psi_{s}(x)$ is given by the solution of the Hamilton-Jacobi-Bellman equation (Fleming and Soner [6]):

$$
0=\max _{p}\left[-\pi_{1}(x-\theta)^{2}-\pi_{2}(p-\bar{p})^{2}+\mathcal{A} \psi_{s}(p, x)\right]
$$

where

$$
\mathcal{A} \psi_{s}(p, x) \equiv \frac{\partial \psi_{s}}{\partial s}+\mu(s) q(s, p) \frac{\partial \psi_{s}}{\partial x}+\frac{1}{2}(\sigma(s))^{2} \frac{\partial^{2} \psi_{s}}{\partial x^{2}}
$$

and $\psi_{T}(x)=-\pi_{1}(x-\theta)^{2}$.

Theorem 3.1. Let $W_{s}(x)$ be the solution of Equation (3.1). The minimum of the expected cost from s to $T$ can be obtained by

$$
W_{s}(x)=Q(s) x^{2}+R(s) x+M(s)
$$

\footnotetext{
${ }^{\ddagger}$ We distinguish between $p_{0}$ and $\bar{p}$. Since the price fluctuates, depending on the real-time demand under the dynamic pricing policy, the customers will be dissatisfied with the price if it is much higher than the historical average price $p_{0}$. To obtain the customers' consent for the dynamic pricing, the seller needs to seek an appropriate target price $\bar{p}$ with consideration of the price fluctuations over the entire period. For this reason, we distinguish the historical average price $\bar{p}$ from the traditional price $p_{0}$.
} 
where $g_{1}^{ \pm}=\mu_{2} \pm \mu_{1} \eta, g_{2}^{ \pm}=1 \pm \sqrt{\pi_{1} / \pi_{2}} a_{2} \mu_{2}$,

$$
\begin{aligned}
& \eta=\frac{a_{1}\left(g_{2}^{-} y_{2}(\hat{t})-g_{2}^{+}\right)}{a_{2}\left(g_{2}^{-} y_{2}(\hat{t})+g_{2}^{+}\right)} \\
& Q(s)= \begin{cases}\frac{\sqrt{\pi_{1} \pi_{2}}\left(g_{1}^{+} y_{1}(s)-g_{1}^{-}\right)}{a_{1} \mu_{1}\left(g_{1}^{+} y_{1}(s)+g_{1}^{-}\right)}, & \text {if } 0 \leq s \leq \hat{t}, \\
\frac{\sqrt{\pi_{1} \pi_{2}}\left(g_{2}^{-} y_{2}(s)-g_{2}^{+}\right)}{a_{2} \mu_{2}\left(g_{2}^{-} y_{2}(s)+g_{2}^{+}\right)}, & \text {if } \hat{t} \leq s \leq T,\end{cases} \\
& R(s)= \begin{cases}\frac{2 \sqrt{y_{1}(s)}}{g_{1}^{+} y_{1}(s)+g_{1}^{-}}\left[\frac{\sqrt{\pi_{2}}}{a_{1} \mu_{1} \sqrt{\pi_{1}}}\left\{\pi_{1} \theta \zeta_{1}^{-}(s)+\frac{\sqrt{\pi_{1} \pi_{2}}}{a_{1}} q(s, \bar{p}) \zeta_{1}^{+}(s)\right\}+\mu_{2} R_{2}(\hat{t})\right], & \text { if } 0 \leq s \leq \hat{t}, \\
\frac{2 \sqrt{y_{2}(s)}}{g_{2}^{-} y_{2}(s)+g_{2}^{+}}\left[\frac{\sqrt{\pi_{2}}}{a_{2} \mu_{2} \sqrt{\pi_{1}}}\left\{\pi_{1} \theta \zeta_{2}^{-}(s)+\frac{\sqrt{\pi_{1} \pi_{2}}}{a_{2}} q(s, \bar{p}) \zeta_{2}^{+}(s)\right\}+2 \theta \pi_{1}\right], & \text { if } \hat{t} \leq s \leq T,\end{cases} \\
& M(s)= \begin{cases}-\pi_{1} \theta^{2}(1+T-s)+\int_{s}^{\hat{t}} E_{1}(u) d u+\int_{\hat{t}}^{T} E_{2}(u) d u, & \text { if } 0 \leq s \leq \hat{t}, \\
-\pi_{1} \theta^{2}(1+T-s)+\int_{s}^{T} E_{2}(u) d u, & \text { if } \hat{t} \leq s \leq T,\end{cases} \\
& E(s)=\frac{1}{4 \pi_{2}} a_{s}^{2}(\mu(s))^{2} R^{2}(s)+q(s, \bar{p}) \mu(s) R(s)+(\sigma(s))^{2} Q(s), \\
& y(s)= \begin{cases}\exp \left\{-2 \sqrt{\frac{\pi_{1}}{\pi_{2}}} a_{1} \mu_{1}(\hat{t}-s)\right\}, & \text { if } 0 \leq s \leq \hat{t} \\
\exp \left\{-2 \sqrt{\frac{\pi_{1}}{\pi_{2}}} a_{2} \mu_{2}(T-s)\right\}, & \text { if } \hat{t} \leq s \leq T\end{cases} \\
& \zeta^{ \pm}(s)= \begin{cases}g_{1}^{+}\left(1-\sqrt{y_{1}(s)}\right) \pm g_{1}^{-}\left(1-\frac{1}{\sqrt{y_{1}(s)}}\right), & \text { if } 0 \leq s \leq \hat{t}, \\
g_{2}^{-}\left(1-\sqrt{y_{2}(s)}\right) \pm g_{2}^{+}\left(1-\frac{1}{\sqrt{y_{2}(s)}}\right), \quad \text { if } \hat{t} \leq s \leq T .\end{cases}
\end{aligned}
$$

where the indexes $i=1$ and $i=2$ in the functions $R(s), E(s)$, and $y(s)$ denote the functions for $0 \leq s \leq \hat{t}$ and $\hat{t} \leq s \leq T$, respectively. In addition, the optimal price when the demand is $x$ at time $s$ is given by

$$
\hat{p}_{s}(x)=\bar{p}+\frac{1}{2 \pi_{2}} a_{s} \mu(s)(2 Q(s) x+R(s)) .
$$

Proof. See Appendix 6.1.

The optimal price in Equation (3.11) is the target price plus a term which depends on the demand level and the distance from the horizon time $T$. The next proposition provides some analytical properties for the optimal price.

Proposition 3.1. For any $0 \leq s \leq T$, we have

(i) $\hat{p}_{s}(x)$ is increasing in $x \geq 0$.

(ii) If $\bar{p}+\left(1 /\left(2 \pi_{2}\right)\right) a_{s} \mu(s) R(s) \geq 0$, then $\hat{p}_{s}(x) \geq 0$ for $x \geq 0$.

(iii) $\hat{p}_{s}(x)$ is decreasing in $\theta$.

Proof. (i) From Equations (2.2), (2.4) and Lemma 6.1(ii), we have $a_{s} \mu(s)<0$ and $Q(s)<0$ for all $s \in[0, T]$. Thus, $\hat{p}_{s}(x)$ is increasing in $x$.

(ii) From Lemma 6.1(iv), we have $R_{2}(s) \geq 0$. However, the sign of $R_{1}(s)$ is ambiguous. By part (i), if $\hat{p}_{s}(0) \geq 0$, then we have $\hat{p}_{s}(x) \geq 0$ for $x \geq 0$.

(iii) Since $y_{1}(s)>0, a_{1} \mu_{1}<0, g_{1}^{+} y_{1}(s)+g_{1}^{-}<0$ and $\zeta_{1}^{-}(s)>0$ by Lemma $6.1\left(\right.$ iv), $R_{1}(s)$ is increasing in $\theta$. Therefore, $\hat{p}_{s}(x)$ is decreasing in $\theta$. 
These properties have intuitive interpretations. Part (i) implies that the optimal price increases with the demand level. Part (ii) provides the condition for which the price function $\hat{p}_{s}(x)$ is positive. If the condition does not hold, the price is negative when the demand is relatively low. For example, when the electricity demand is low on a windy night, the supply of electricity generated by wind power could exceed the demand. In such a case, the firm pays a fee to have the excess electricity taken up to avoid shutting down their power generation. The fee can be considered as a negative price. Part (iii) means that when the target level $\theta$ is set too high, the optimal price decreases in order to bring demand up to the target level.

Applying the optimal price, the demand process satisfies the following SDE:

$$
\begin{aligned}
d X_{s} & =\mu(s) q\left(s, \hat{p}_{s}\right) d s+\sigma(s) d w_{s} \\
& =\left\{\lambda_{1}(s) X_{s}+\lambda_{2}(s)\right\} d s+\sigma(s) d w_{s},
\end{aligned}
$$

where

$$
\begin{aligned}
\lambda_{1}(s) & =\frac{1}{\pi_{2}}\left(a_{s} \mu(s)\right)^{2} Q(s), \\
\lambda_{2}(s) & =\mu(s)\left[a_{s}\left(\frac{1}{2 \pi_{2}} R(s) a_{s} \mu(s)+\bar{p}-p_{0}\right)+1\right] .
\end{aligned}
$$

This SDE permits a unique strong solution $X_{s}$, which is defined as follows (Theorem 6.14 of Yong and Zhou [7]):

$$
X_{s}=e^{Y_{s}}\left\{x_{0}+\int_{0}^{s} \lambda_{2}(u) e^{-Y_{u}} d u+\int_{0}^{s} \sigma(u) e^{-Y_{u}} d w_{u}\right\}
$$

where $X_{0}=x_{0}$ and

$$
Y_{s} \equiv \int_{0}^{s} \lambda_{1}(u) d u \equiv \begin{cases}Y_{1}(s), & \text { if } 0 \leq s \leq \hat{t} \\ Y_{2}(s), & \text { if } \hat{t} \leq s \leq T\end{cases}
$$

and

$$
\begin{aligned}
& Y_{1}(s)=\log \left(\frac{g_{1}^{+} y_{1}(s)+g_{1}^{-}}{g_{1}^{+} y_{1}(0)+g_{1}^{-}}\right)-\sqrt{\frac{\pi_{1}}{\pi_{2}}} a_{1} \mu_{1} s, \\
& Y_{2}(s)=Y_{1}(\hat{t})+\log \left(\frac{g_{2}^{-} y_{2}(s)+g_{2}^{+}}{g_{2}^{-} y_{2}(\hat{t})+g_{2}^{+}}\right)-\sqrt{\frac{\pi_{1}}{\pi_{2}}} a_{2} \mu_{2}(s-\hat{t}) .
\end{aligned}
$$

The demand $X_{s}$ is a normally distributed random variable, and its expectation and variance are given by

$$
\begin{aligned}
E\left[X_{s}\right] & =e^{Y_{s}}\left\{x_{0}+\int_{0}^{s} \lambda_{2}(u) e^{-Y_{u}} d u\right\}, \\
\operatorname{Var}\left(X_{s}\right) & =e^{2 Y_{s}} \int_{0}^{s}(\sigma(u))^{2} e^{-2 Y_{u}} d u .
\end{aligned}
$$

Here, we define $R(s)$ as $R(s)=I(s) \bar{p}+J(s)$, where

$$
\begin{aligned}
& I(s)= \begin{cases}\frac{2 \sqrt{y_{1}(s)}}{g_{1}^{+} y_{1}(s)+g_{1}^{-}}\left[\frac{\pi_{2}}{a_{1} \mu_{1}} \zeta_{1}^{+}(s)+\mu_{2} I_{2}(\hat{t})\right], & \text { if } 0 \leq s \leq \hat{t}, \\
\frac{2 \sqrt{y_{2}(s)}}{g_{2}^{-} y_{2}(s)+g_{2}^{+}} \frac{\pi_{2}}{a_{2} \mu_{2}} \zeta_{2}^{+}(s), & \text { if } \hat{t} \leq s \leq T,\end{cases} \\
& J(s)= \begin{cases}\frac{2 \sqrt{y_{1}(s)}}{g_{1}^{+} y_{1}(s)+g_{1}^{-}}\left[\frac{\sqrt{\pi_{2}}}{a_{1} \mu_{1} \sqrt{\pi_{1}}}\left\{\pi_{1} \theta \zeta_{1}^{-}(s)+\frac{\sqrt{\pi_{1} \pi_{2}}}{a_{1}}\left(1-a_{1} p_{0}\right) \zeta_{1}^{+}(s)\right\}+\mu_{2} J_{2}(\hat{t})\right], & \text { if } 0 \leq s \leq \hat{t}, \\
\frac{2 \sqrt{y_{2}(s)}}{g_{2}^{-} y_{2}(s)+g_{2}^{+}}\left[\frac{\sqrt{\pi_{2}}}{a_{2} \mu_{2} \sqrt{\pi_{1}}}\left\{\pi_{1} \theta \zeta_{2}^{-}(s)+\frac{\sqrt{\pi_{1} \pi_{2}}}{a_{2}}\left(1-a_{2} p_{0}\right) \zeta_{2}^{+}(s)\right\}+2 \theta \pi_{1}\right], & \text { if } \hat{t} \leq s \leq T .\end{cases}
\end{aligned}
$$


Lemma 3.1. $I(s)>0$ for all $s \in[0, T]$.

Proof. Since $a_{2} \mu_{2}<0, \zeta_{2}^{+}(s)<0$ and $g_{2}^{-} y_{2}(s)+g_{2}^{+}>0$, we have $I_{2}(s)>0$ for all $s$. Moreover, by $g_{1}^{+} y_{1}(s)+g_{1}^{-}<0$ and $\zeta_{1}^{+}>0$, we obtain $I_{1}(s)>0$ for all $s$.

Proposition 3.2. If $a_{2} \mu_{2} \leq a_{1} \mu_{1}$ holds, then $\hat{p}_{s}(x)$ is increasing in $\bar{p}$, and $E\left[X_{s}\right]$ is decreasing in $\bar{p}$.

Proof. For $s \in[0, \hat{t}]$, by Equations (3.21) and (3.10), we have

$$
\begin{aligned}
\frac{\partial \hat{p}_{s}(x)}{\partial \bar{p}} & =\frac{1}{2 \pi_{2}} a_{1} \mu_{1} I_{1}(s)+1 \\
& =\frac{2 \sqrt{y_{1}(s)}}{g_{1}^{+} y_{1}(s)+g_{1}^{-}}\left[\mu_{2}+\frac{2 a_{1} \mu_{1}}{a_{2}} \frac{y_{2}(\hat{t})}{g_{2}^{-} y_{2}(\hat{t})+g_{2}^{+}}-\frac{a_{1} \mu_{1}}{a_{2}}\right] .
\end{aligned}
$$

Since $g_{1}^{+} y_{1}(s)+g_{1}^{-}<0, g_{2}^{-} y_{2}(\hat{t})+g_{2}^{+}>0$ and $a_{2} \mu_{2} \leq a_{1} \mu_{1}$, we have $\frac{\partial \hat{p}_{s}(x)}{\partial \bar{p}}>0$. For $s \in[\hat{t}, T]$, we obtain

$$
\frac{\partial \hat{p}_{s}(x)}{\partial \bar{p}}=\frac{1}{2 \pi_{2}} a_{2} \mu_{2} I_{2}(s)+1=\frac{2 \sqrt{y_{2}(s)}}{g_{2}^{-} y_{2}(s)+g_{2}^{+}}>0 .
$$

Next, we show that the expected value

$$
\frac{\partial}{\partial \bar{p}} E\left[X_{s}\right]=e^{Y_{s}} \int_{0}^{s} \frac{\partial}{\partial \bar{p}} \lambda_{2}(u) e^{-Y_{u}} d u
$$

Since $\frac{\partial}{\partial \bar{p}} \lambda_{2}(u)=a_{u} \mu(u)\left(\frac{1}{2 \pi_{2}} a_{u} \mu(u) I(u)+1\right)<0$, we obtain $\frac{\partial}{\partial \bar{p}} E\left[X_{s}\right]<0$.

The assumption $a_{2} \mu_{2} \leq a_{1} \mu_{1}$ implies that customers are more sensitive to prices in period $[\hat{t}, T]$ than in period $[0, \hat{t}]$. As the target price $\bar{p}$ increases due to a rapidly increasing commodity price, the optimal price increases and the expected demand decreases.

Proposition 3.3. Let $X_{s}^{c}$ be the demand at time $s$ when a fixed price $p_{0}$ is applied throughout the selling period $[0, T]$. The variance of the peak demand with dynamic pricing is less than that of fixed pricing: $\operatorname{Var}\left[X_{\hat{t}}\right]<\operatorname{Var}\left[X_{\hat{t}}^{c}\right]$.

Proof. By Equation (3.20) and $\operatorname{Var}\left(X_{\hat{t}}^{c}\right)=\sigma^{2} \hat{t}$, we have

$$
\frac{\operatorname{Var}\left(X_{\hat{t}}\right)}{\operatorname{Var}\left(X_{\hat{t}}^{c}\right)}=\frac{1}{\hat{t}} \int_{0}^{\hat{t}} e^{2\left(Y_{1}(\hat{t})-Y_{1}(u)\right)} d u
$$

Putting (3.17) into Equation (3.26), we obtain

$$
\begin{aligned}
\frac{\operatorname{Var}\left(X_{\hat{t}}\right)}{\operatorname{Var}\left(X_{\hat{t}}^{c}\right)} & =\frac{1}{\hat{t}} \int_{0}^{\hat{t}}\left(\frac{2 \mu_{2}}{g_{1}^{+} y_{1}(u)+g_{1}^{-}}\right)^{2} e^{-2 \sqrt{\frac{\pi_{1}}{\pi_{2}}} a_{1} \mu_{1}(\hat{t}-u)} d u \\
& =\frac{\mu_{2}}{a_{1} \mu_{1} \hat{t}} \sqrt{\frac{\pi_{2}}{\pi_{1}}} \frac{1-y_{1}(0)}{g_{1}^{+} y_{1}(0)+g_{1}^{-}} \\
& \approx \frac{2 \mu_{2}}{g_{1}^{+} y_{1}(0)+g_{1}^{-}} \\
& <1 .
\end{aligned}
$$

The third equation comes from Taylor expansion for $y_{1}(0) \approx 1-2 \sqrt{\pi_{1} / \pi_{2}} a_{1} \mu_{1} \hat{t}$. The last inequality is obtained by $y_{1}(0)>1, g_{1}^{+}<0$ and $g_{1}^{+} y_{1}(0)<g_{1}^{+}+g_{1}^{-}=2 \mu_{2}<0$. 
Proposition 3.4. Let $\bar{X} \equiv \int_{0}^{T} X_{s} d s$ be the cumulative demand in time interval $[0, T]$ at time $t$ when the optimal price is used. Then, $\bar{X}$ follows a normal distribution: $\bar{X} \sim N\left(\tilde{\mu}, \tilde{\sigma}^{2}\right)$, where

$$
\begin{aligned}
\tilde{\mu} & \equiv \int_{0}^{T} E\left[X_{s}\right] d s \\
\tilde{\sigma}^{2} & \equiv \int_{0}^{T} \int_{0}^{T} e^{Y_{u}+Y_{v}} \min \left\{\int_{0}^{u}(\sigma(m))^{2} e^{-2 Y_{m}} d m, \int_{0}^{v}(\sigma(m))^{2} e^{-2 Y_{m}} d m\right\} d u d v .
\end{aligned}
$$

Proof. See Appendix 6.2.

Proposition 3.5. If $a_{2} \mu_{2} \leq a_{1} \mu_{1}$ holds, then $\tilde{\mu}$ is also decreasing in $\bar{p}$.

Proof. Since $E\left(X_{s}\right)$ is decreasing in $\bar{p}$ by Proposition 3.2, this result directly follows from Equation 3.27 .

\section{Numerical Examples}

In this section, we investigate the behavior of the optimal price and the effect of dynamic pricing on peak demand using numerical examples. We use the electricity pricing decision as the context for our examples. The time horizon is set as $T=1$. Demand data were obtained from the Tokyo Electric Power Company Holdings (TEPCO, [8]) which reports electricity usage at 5-minute intervals. We fitted our model parameters to the electricity usage data from 21 August 2012, using the method of moments to estimate the values $\mu_{1}$, $\mu_{2}, \sigma_{1}$, and $\sigma_{2}$ for the coefficients of the demand process in Equation (2.1). We discretized the time interval $[0,1]$ into $L$ time periods of length $\Delta t=1 / L$. Since the data are reported at 5-minute intervals, the time interval (i.e., one day) is divided into $L=288$ periods. The peak time is set as $\hat{t}=0.5$, and the length of the time intervals $[0, \hat{t}]$ and $[\hat{t}, 1]$ are $L_{1}=144$ and $L_{2}=144$, respectively. The coefficient of the demand process $\mu(s)$ is defined by Equation (2.2), and $\sigma(s)$ is defined by

$$
\sigma(s)= \begin{cases}\sigma_{1} \geq 0, & \text { if } 0 \leq s<\hat{t} \\ \sigma_{2} \leq 0, & \text { if } \hat{t} \leq s \leq \bar{T}\end{cases}
$$

The demand process for sub-interval $k=1,2$ was discretized as follows:

$$
X_{n+1}=X_{n}+\mu_{k} \Delta t+\sigma_{k} \sqrt{\Delta t} Z_{n}, \quad n=0,1, \ldots, L_{k},
$$

where $Z_{n}$ is an independent and identically distributed standard normal random variable. Thus, we have $E\left[X_{n+1}-X_{n}\right]=\mu_{k} \Delta t$ and $\operatorname{Var}\left(X_{n+1}-X_{n}\right)=\sigma_{k}^{2} \Delta t, k=1,2$. Let $\hat{\mu}_{j, k}$ be the $j$-th sample moment for sub-interval $k: \hat{\mu}_{j, k}=(1 / N) \sum_{n=1}^{N} X_{n}^{j, k}$, where $N$ is the sample size. By using the method of moments, we have $\mu_{k}=\hat{\mu}_{1, k} / \Delta t$ and $\sigma_{k}=\sqrt{\left(\hat{\mu}_{2, k}-\hat{\mu}_{1, k}^{2}\right) / \Delta t}$.

Table 1 summarizes the estimated parameters and the set of baseline sensitivity parameters. The demand data and the sample path derived by model (2.1) with its estimated parameters are shown in Figure 1. Figure 2 shows the optimal price which is derived by Equation (3.11), and the corresponding demand is shown in Figure 1.

We can see that the peak demand for the dynamic pricing model is smaller than that of the fixed pricing model. As shown in Figure 2, the optimal price rises ahead of the peak time. This behavior is realistic, as suppliers constrain demand by increasing the price before peak demand. In Figure 3, we show the average sample paths of the optimal price. We 
recorded all the trajectories of the selling prices along the simulated sample paths and then calculated their means. The average price also rises ahead of the peak time. To investigate the cause of this phenomenon, we consider the isopleths of the optimal price $\hat{p}_{s}(x)$ for $[0, \hat{t}]$ in Figure 4. We see that the optimal price increases as the demand becomes larger and decreases as the time approaches the peak time. Thus, the decrease of the optimal price due to the time elapsed would lead to an increase in the price ahead of the peak time.

Next, we investigate the effect of the parameters on the peak demand for the dynamic pricing model, and compare the peak demand of the dynamic pricing model with that of the fixed pricing model. Figure 5 shows the expected peak demand with respect to the price response factor $a \equiv\left|a_{1}\right|=\left|a_{2}\right|$. We observe that the expected peak-demand for the dynamic pricing is decreasing with the price response factor. This implies that dynamic pricing is effective when the customer is sensitive to price. However, as shown in Figure 5, if the customer is not sensitive to price, then the fixed pricing approach is better.

Figure 6 shows the variance in the peak demand with respect to the rate for the volatility $\left(\alpha \sigma_{1}, \alpha \sigma_{2}\right)$. The variance tends to increase with the volatility. This indicates that the variance of the peak demand under the dynamic pricing policy is smaller than the variance under the fixed pricing policy.

Figures 7 and 8 show the mean and variance of the peak demand as a function of the stability factor $\pi_{1}$. As expected, the mean demand decreases as the stability factor increases.

Figure 9 shows the expected cumulative demand under dynamic and fixed pricing policies with respect to $a$. We can see that dynamic pricing is an effective method for reducing electricity consumption when the customers are sensitive to the price.

\section{Conclusions}

In this paper, we consider a dynamic pricing model for stabilizing the peak demand in which the dynamics of the demand follow a stochastic differential equation. The closed form of the optimal pricing policy is derived and some analytical properties are investigated. In particular, we show that dynamic pricing not only reduces peak demand but also mitigates the fluctuations in the peak demand. Moreover, it is shown that a fixed pricing policy is effective when the customers are not sensitive to prices.

For future research, we wish to extend our model to include a limit on the supply capacity and to take into account the multiple supply sources. For instance, an electricity company generates electricity from several sources (e.g., nuclear power, natural gas, coal, and solar energy). These sources have different constraints on their availability and cost. Thus, we

Table 1: Summary of the model parameters and parameter estimates for the demand model fitted to the electricity data from 22/8/2012

\begin{tabular}{lclc}
\hline \hline Stochastic Process Parameters & \multicolumn{3}{c}{ Operating Parameters } \\
\hline Initial demand $\left(x_{0}\right)$ & 25 & Price response factor in $[0, \hat{t}]\left(a_{1}\right)$ & -0.07 \\
Drift rate in $[0, \hat{t}]\left(\mu_{1}\right)$ & 43.98 & Price response factor in $[\hat{t}, T]\left(a_{2}\right)$ & 0.07 \\
Drift rate in $[\hat{t}, T]\left(\mu_{2}\right)$ & -40 & Conventional fixed price $\left(p_{0}\right)$ & 24 \\
Volatility in $[0, \hat{t}]\left(\sigma_{1}\right)$ & 3.957 & Stability factor for demand $\left(\pi_{1}\right)$ & 5 \\
Volatility in $[\hat{t}, T]\left(\sigma_{2}\right)$ & 2.755 & Stability factor for price $\left(\pi_{2}\right)$ & 1 \\
& & Target price level $(\bar{p})$ & 24 \\
& Target demand level $(\theta)$ & 36 \\
\hline \hline
\end{tabular}




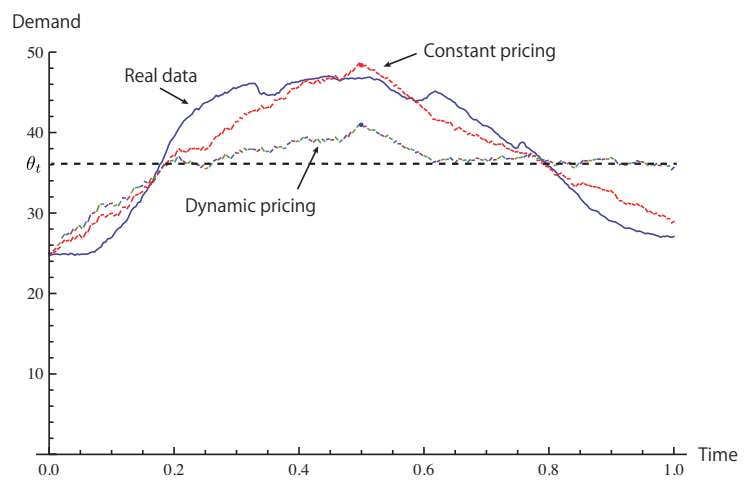

Figure 1: Daily demand curves on $21 \mathrm{Au}-$ gust 2012 and the sample demand path based on our model. $t=0$ corresponds to 4 am. (Unit: GW)

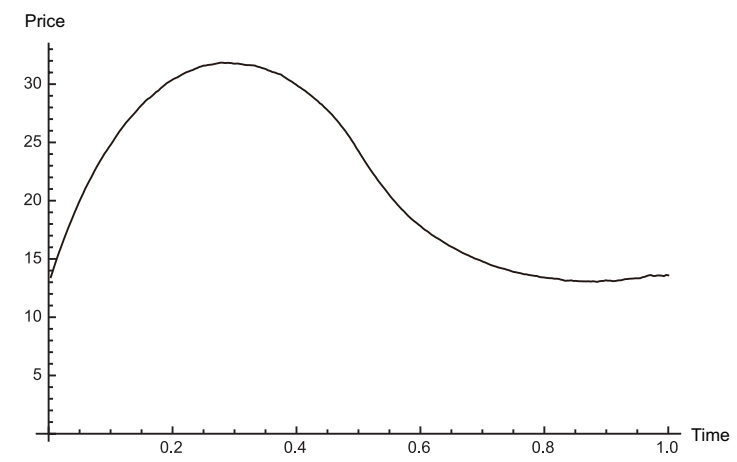

Figure 3: Average sample path of the optimal price with respect to time

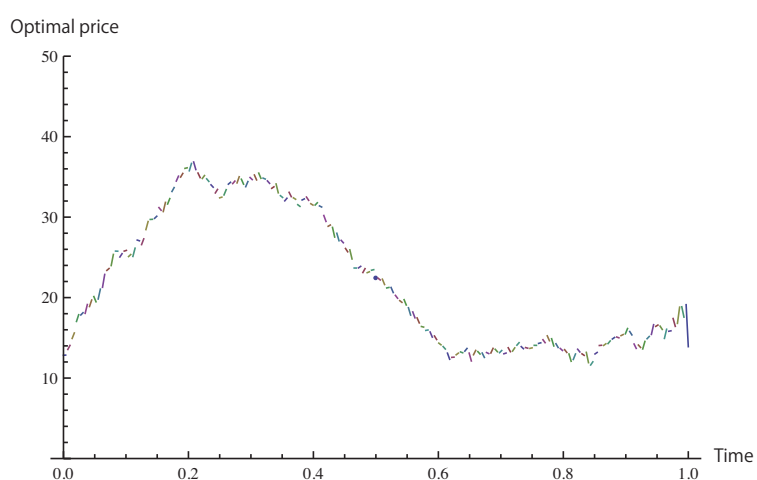

Figure 2: Optimal price corresponding to the sample demand path (yen)

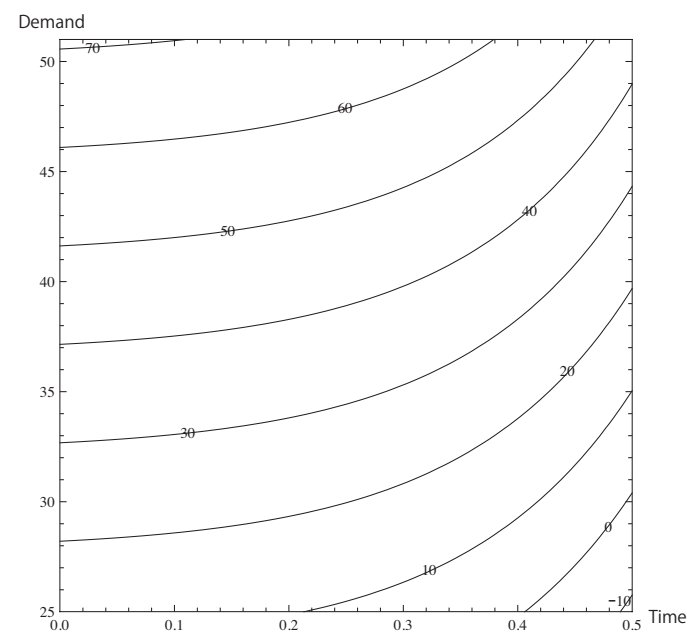

Figure 4: Optimal price with respect to time and demand

hope it will be an interesting topic to extend our models to a setting with multiple suppliers, incorporating stochastic commodity prices and operational constraints.

Acknowledgment We are very grateful to the anonymous referee for his or her insightful and detailed comments that substantially improved the quality of the paper. This paper is supported in part by a JSPS Grant-in-Aid for Young Scientists (B) (26870643).

\section{References}

[1] S. Yousefi, M.P. Moghaddam, and V.J. Majd: Optimal real time pricing in an agentbased retail market using a comprehensive demand response model. Energy, 36 (2011), 5716-5727.

[2] M. Doostizadeh and H. Ghasemi: A day-ahead electricity pricing model based on smart metering and demand-side management. Energy, 46 (2012), 221-230.

[3] M. Roozbehani, M.A. Dahleh, and S.K. Mitter: Dynamic pricing and stabilization of supply and demand in modern electric power grids. Proceedings of the First IEEE International Conference on Smart Grid Communications, (2010), 543-548. 


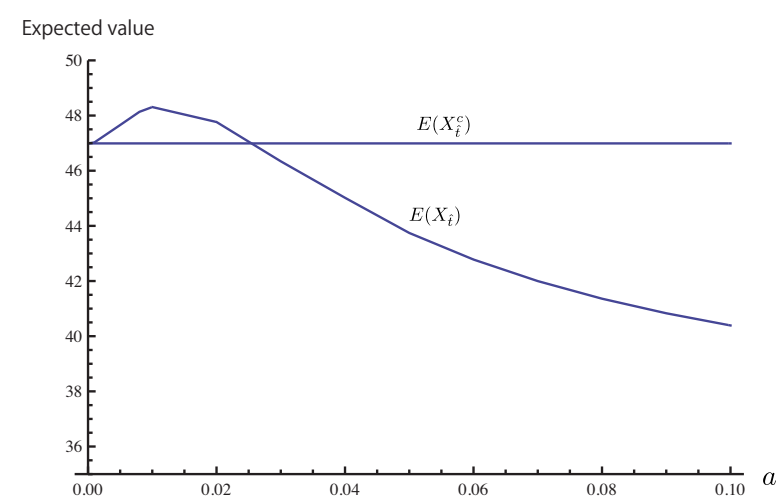

Figure 5: Expected peak demand with respect to $a$

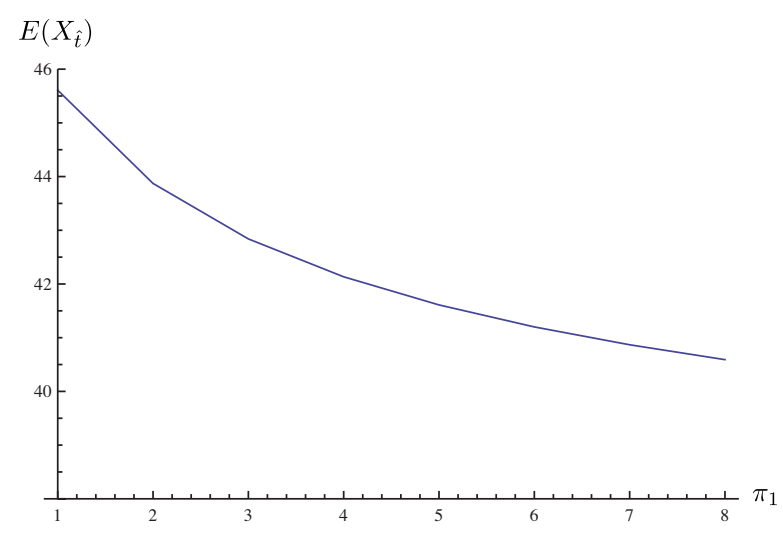

Figure 7: Expected peak demand with respect to $\pi_{1}$

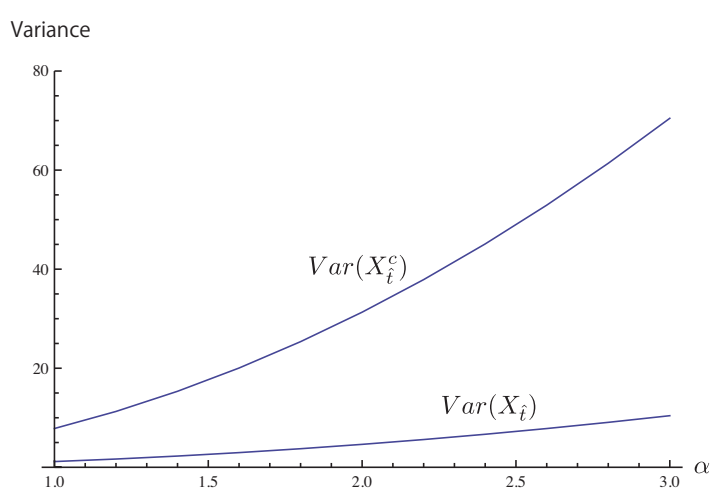

Figure 6: Variance of peak demand with respect to $a$

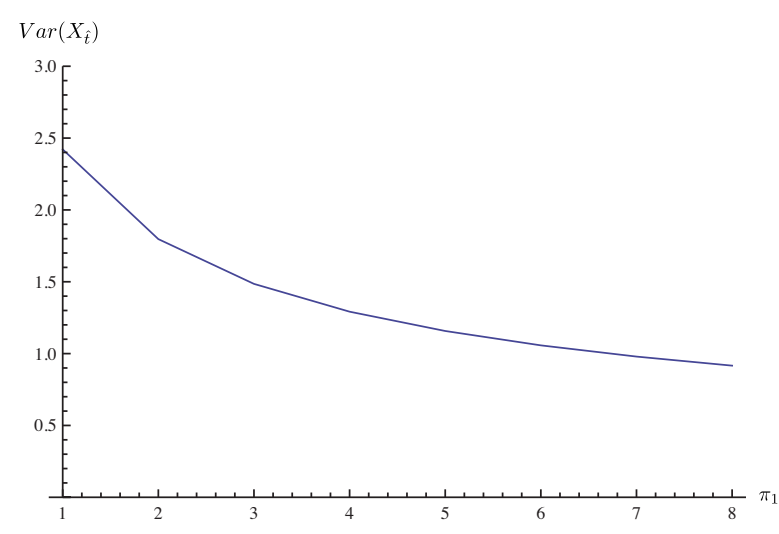

Figure 8: Variance of peak demand with respect to $\pi_{1}$

[4] A. Bensoussan, S.P. Sethi, R. Vickson, and N. Derzko: Stochastic production planning with production constraints. SIAM Journal on Control and Optimization, 22 (1984), 920-935.

[5] S.P. Sethi and G.L. Thompson: Optimal Control Theory, Applications to Management Science and Economics (Kluwer Academic Publishers, Norwell, 2000).

[6] W.H. Fleming and H.M. Soner: Controlled Markov Processes and Viscosity Solutions (Springer-Verlag, New York, 1993).

[7] J. Yong and Z.Y. Zhou: Stochastic Controls: Hamiltonian Systems and HJB Equations (Springer-Verlag, New York, 1999).

[8] Tokyo Electric Power Company Holdings, Inc.: http://www.tepco.co.jp/forecast/indexj.html, (August 30, 2012). 


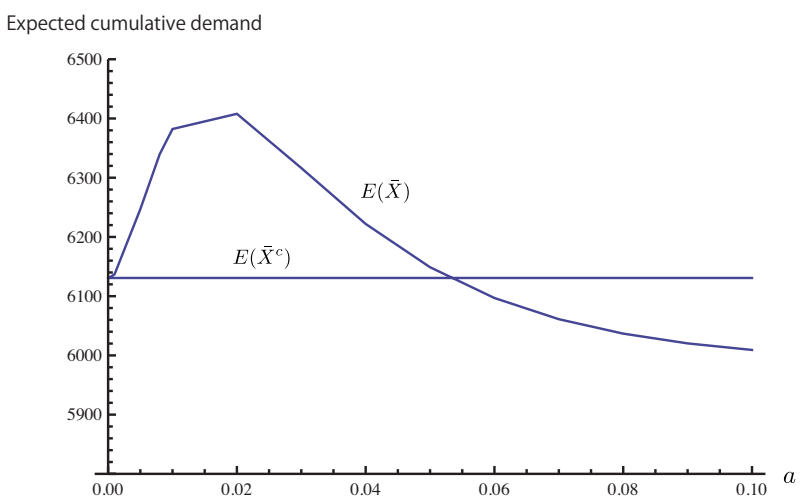

Figure 9: Expected cumulative demand with respect to $a$

\section{Appendix}

\subsection{Proof of Theorem 3.1}

Proof. Define the function

$$
w(p)=-\pi_{2}(p-\bar{p})^{2}+\mu(s) q(s, p) \frac{\partial W_{s}}{\partial x} .
$$

We have

$$
w^{\prime}(p)=-2 \pi_{2}(p-\bar{p})+\mu(s) a_{s} \frac{\partial W_{s}}{\partial x}
$$

and $w^{\prime \prime}(p)=-2 \pi_{2}<0$. Thus, $w(p)$ is concave in $p$, and the first-order condition leads to

$$
\hat{p}_{s}(x)=\bar{p}+\frac{1}{2 \pi_{2}} a_{s} \mu(s) \frac{\partial W_{s}}{\partial x} .
$$

Substituting Equation (6.3) into Equation (3.1), we obtain

$$
-\pi_{1}(x-\theta)^{2}+\frac{1}{4 \pi_{2}} a_{s}^{2} \mu^{2}(s)\left(\frac{\partial W_{s}}{\partial x}\right)^{2}+\frac{\partial W_{s}}{\partial s}+\mu(s) q(s, \bar{p}) \frac{\partial W_{s}}{\partial x}+\frac{1}{2}(\sigma(s))^{2} \frac{\partial^{2} W_{s}}{\partial x^{2}}=0 .
$$

Since the functions $\mu(s)$ and $a_{s}$ are not continuous with respect to $s$, we try a function of the form

$$
W_{s}(x)= \begin{cases}Q_{1}(s) x^{2}+R_{1}(s) x+M_{1}(s), & \text { if } 0 \leq s \leq \hat{t} \\ Q_{2}(s) x^{2}+R_{2}(s) x+M_{2}(s), & \text { if } \hat{t} \leq s \leq T .\end{cases}
$$

Define $W_{s}(x)=W_{s}^{1}(x)$ for $0 \leq s \leq \hat{t}$ and $W_{s}(x)=W_{s}^{2}(x)$ for $\hat{t} \leq s \leq T$. Then our objective is to solve Equation (6.4) with boundary conditions $W_{\hat{t}}^{1}(x)=W_{\hat{t}}^{2}(x)$ and $W_{T}^{2}(x)=$ $-\pi_{1}(x-\theta)^{2}$.

Putting Equation (6.5) into Equation (6.4) we have

$$
\begin{aligned}
\frac{d Q(s)}{d s} & =\pi_{1}-\frac{1}{\pi_{2}} a_{s}^{2} \mu^{2}(s) Q^{2}(s) \\
\frac{d R(s)}{d s} & =-2 \pi_{1} \theta-\frac{1}{\pi_{2}} a_{s}^{2} \mu^{2}(s) Q(s) R(s)-2 Q(s) q(s, \bar{p}) \mu(s), \\
\frac{d M(s)}{d s} & =\pi_{1} \theta^{2}-\frac{1}{4 \pi_{2}} a_{s}^{2} \mu^{2}(s) R^{2}(s)-q(s, \bar{p}) \mu(s) R(s)-\sigma^{2}(s) Q(s) .
\end{aligned}
$$


First, we solve Equation (6.6). The solution is given by

$$
Q(s)=\frac{1}{\mu(s)} \frac{C e^{2 s \sqrt{b_{1} b_{2}} \mu(s)}-1}{C e^{2 s \sqrt{b_{1} b_{2}} \mu(s)}+1} \sqrt{\frac{b_{1}}{b_{2}}}
$$

where $b_{1}=\pi_{1}, b_{2}=a_{s}^{2} / \pi_{2}$ and $C$ is an arbitrary constant. For $\hat{t} \leq s \leq T$, the boundary condition gives $Q_{2}(T)=-\pi_{1}$. Thus, we have

$$
C=\frac{1-\sqrt{\frac{\pi_{1}}{\pi_{2}}} a_{2} \mu_{2}}{1+\sqrt{\frac{\pi_{1}}{\pi_{2}}} a_{2} \mu_{2}} \exp \left\{-2 \sqrt{\frac{\pi_{1}}{\pi_{2}}} a_{2} \mu_{2} T\right\} .
$$

This gives $Q_{2}(s)$ in Equation (3.5). For $0 \leq s \leq \hat{t}$, the boundary condition gives $Q(\hat{t})=$ $Q_{2}(\hat{t})$. From Equation (6.9), we have

$$
C=\frac{\mu_{1} \eta+\mu_{2}}{\mu_{2}-\mu_{1} \eta} \exp \left\{-2 \sqrt{\frac{\pi_{1}}{\pi_{2}}} a_{1} \mu_{1} \hat{t}\right\}
$$

where $\eta=a_{1}\left(g_{2}^{-} y_{2}(\hat{t})-g_{2}^{+}\right) / a_{2}\left(g_{2}^{-} y_{2}(\hat{t})+g_{2}^{+}\right)$and $g_{2}^{ \pm}=1 \pm \sqrt{\pi_{1} / \pi_{2}} a_{2} \mu_{2}$. Here, $y_{2}(\cdot)$ is given by Equation (3.9). By substituting it into (6.9), we obtain $Q_{1}(s)$ in Equation (3.5).

Next, we solve Equation (6.7). Suppose that the boundary condition is $R(\tilde{T})=C_{0}$. Then, we can obtain the solution as follows:

$$
R(s)=e^{\int_{s}^{\tilde{T}} \frac{1}{\pi_{2}} \mu_{u}^{2} a_{u} Q(u) d u}\left\{\int_{s}^{\tilde{T}}\left(2 \pi_{1} \theta+2 Q(u) q(\bar{p}) \mu(u)\right) e^{-\int_{u}^{\tilde{T}} \frac{1}{\pi_{2}} \mu_{r}^{2} a_{r}^{2} Q(r) d r} d u+C_{0}\right\} .
$$

For $\hat{t} \leq s \leq T$, the boundary condition gives $R_{2}(T)=C_{0}=2 \pi_{1} \theta$. In addition, we have

$$
\begin{aligned}
\int_{u}^{\tilde{T}} \frac{1}{\pi_{2}} \mu_{r}^{2} a_{r}^{2} Q(r) d r & =\frac{1}{\pi_{2}} a_{2}^{2} \mu_{2} \int_{u}^{T} Q_{2}(r) d r \\
& =\log \left(\frac{2}{g_{2}^{-} y_{2}(u)+g_{2}^{+}}\right)-\sqrt{\frac{\pi_{1}}{\pi_{2}}} a_{2} \mu_{2}(T-u)
\end{aligned}
$$

Substituting it into Equation (6.12), we obtain $R_{2}(s)$ in Equation (3.6). For $0 \leq s \leq \hat{t}$, replacing $\tilde{T}$ to $\hat{t}$ and $C_{0}$ to $R_{2}(\hat{t})$ in Equation (6.12), we have $R_{1}(s)$ in Equation (3.6).

Finally, by using the solutions $Q(x)$ and $R(x)$, we can easily obtain the solution of Equation (6.8) for $\hat{t} \leq s \leq T$ as

$$
M_{2}(s)=-\pi_{1} \theta^{2}(1+T-s)+\int_{s}^{T} E_{2}(u) d u,
$$

where

$$
E_{2}(s)=\frac{1}{4 \pi_{2}} a_{2}^{2} \mu_{2}^{2} R_{2}(s)+q(s, \bar{p}) \mu_{2} R_{2}(s)+\sigma_{2}^{2} Q_{2}(s) .
$$

It follows from $M_{1}(\hat{t})=M_{2}(\hat{t})$ that

$$
M_{1}(s)=M_{2}(\hat{t})-\int_{s}^{\hat{t}} E_{1}(u) d u,
$$


where

$$
E_{1}(s)=\frac{1}{4 \pi_{2}} a_{1}^{2} \mu_{1}^{2} R_{1}(s)+q(s, \bar{p}) \mu_{1} R_{1}(s)+\sigma_{1}^{2} Q_{1}(s) .
$$

The optimal price is given by Equation (6.3), and the use of $Q(s)$ and $R(s)$ yields Equation (3.11).

Next lemma will be used to show some analytical properties.

Lemma 6.1. For $0 \leq s \leq T$, we have

(i) $1 \leq y_{i}(s), i=1,2$.

(ii) $Q_{i}(s)<0, i=1,2$.

(iii) $\eta<0$.

(iv) $\zeta_{1}^{ \pm}(s)>0$ and $\zeta_{2}^{ \pm}(s)<0$.

Proof. We only show (i)-(iv) for $i=1$.

(i) Since $y_{1}(s)$ is decreasing in $s$ for $0 \leq s \leq \hat{t}$ and $y_{1}(\hat{t})=1$, we have $1 \leq y_{1}(s)$.

(ii) First, we show that $\eta<0$ which is part (iii). From $a_{2}>0$ and $\mu_{2}<0$, we have $g_{2}^{-}>0$. For $g_{2}^{+}>0$, it follows from part (i) that $g_{2}^{-} y_{2}(s)-g_{2}^{+}>g_{2}^{-}-g_{2}^{+}=-2 \sqrt{\pi_{1} / \pi_{2}} a_{2} \mu_{2}>0$. Moreover, we obtain $g_{2}^{-} y_{2}(s)+g_{2}^{+}>0$. For $g_{2}^{+}<0$, we have $g_{2}^{-} y_{2}(s)-g_{2}^{+}>0$. In addition, $g_{2}^{-} y_{2}(s)+g_{2}^{+}>g_{2}^{-}+g_{2}^{+}=2>0$. Thus, we obtain $\eta<0$. It gives $g_{1}^{+}=\mu_{2}+\mu_{1} \eta<0$. For $g_{1}^{-}>0$, we have $g_{1}^{+} y_{1}(s)-g_{1}^{-}<0$. In addition, we get $g_{1}^{+} y_{1}(s)+g_{1}^{-}<g_{1}^{+}+g_{1}^{-}=2 \mu_{2}<0$. Since $a_{1} \mu_{1}<0$, we have $Q_{1}(s)<0$. For $g_{1}^{-}<0$, we have $g_{1}^{+} y_{1}(s)+g_{1}^{-}<0$. Since $g_{1}^{+} y_{1}(s)-g_{1}^{-}<g_{1}^{+}-g_{1}^{-}=2 \mu_{1} \eta<0$, we have $Q_{1}(s)<0$.

(iv) If $g_{1}^{-}>0$, then $\zeta_{1}^{+}(s)>0$ by part (i). When $g_{1}^{-}<0, \zeta_{1}^{+}(s)$ can be rewritten as $\zeta_{1}^{+}(s)=$ $\left(g_{1}^{+}-g^{-}\left(y_{1}(s)\right)^{-\frac{1}{2}}\right)\left(1-\left(y_{1}(s)\right)^{\frac{1}{2}}\right)$. Here, we see that $g_{1}^{+}-g^{-}\left(y_{1}(s)\right)^{-\frac{1}{2}}<g_{1}^{+}-g_{1}^{-}=2 \mu_{1} \eta<0$. Hence, $\zeta_{1}^{+}(s)>0$. Similarly, we can obtain $\zeta_{1}^{-}(s)>0$.

\subsection{Proof of Proposition 3.4}

Proof. The expectation of $\bar{X}$ is given by $E[\bar{X}]=\int_{0}^{T} E\left[X_{s}\right] d s$. The covariance of $\bar{X}_{s}$ and $\bar{X}_{m}$ is given by

$$
\begin{aligned}
\operatorname{Cov}\left(\bar{X}_{s}, \bar{X}_{m}\right) & =E\left[\bar{X}_{s} \bar{X}_{m}\right]-E\left[\bar{X}_{s}\right] E\left[\bar{X}_{m}\right] \\
& =\int_{0}^{s} \int_{0}^{m} E\left[X_{u} X_{v}\right] d u d v-\tilde{\mu}_{s} \tilde{\mu}_{m} \\
& =\int_{0}^{s} \int_{0}^{m}\left(\operatorname{Cov}\left(X_{u}, X_{v}\right)+E\left(X_{u}\right) E\left(X_{v}\right)\right) d u d v-\tilde{\mu}_{s} \tilde{\mu}_{m} . \\
& =\int_{0}^{s} \int_{0}^{m} e^{Y_{u}+Y_{v}} \min \left\{\int_{0}^{u}(\sigma(m))^{2} e^{-2 Y_{m}} d m, \int_{0}^{v}(\sigma(m))^{2} e^{-2 Y_{m}} d m\right\} d u d v
\end{aligned}
$$

By setting $s=m=T$, we obtain the equation (3.28).

Kimitoshi Sato

Kanagawa University

Faculty of Engineering

3-27-1 Rokkakubashi, Kanagawa-ku, Yokohama, Kanagawa 221-8686, Japan

E-mail: k-sato@kanagawa-u.ac.jp 